\title{
Correction to: Comparative genomics of Alternaria species provides insights into the pathogenic lifestyle of Alternaria brassicae - a pathogen of the Brassicaceae family
}

Sivasubramanian Rajarammohan ${ }^{1,2}$, Kumar Paritosh $^{3}$, Deepak Pental ${ }^{3}$ and Jagreet Kaur ${ }^{1^{*}}$

\section{Correction to: BMC Genomics (2019) 20:1036}

https://doi.org/10.1186/s12864-019-6414-6

Following the publication of this article [1], the authors reported that the captions of Figs. 2 and 3 were published in the incorrect order, whereby they mismatch with their corresponding images. The figures are reproduced in the correct sequence with the correct captions in this Correction article. The original article has been corrected.

\footnotetext{
Author details

'Department of Genetics, University of Delhi, South Campus, New Delhi 110021, India. ${ }^{2}$ Present Address: National Agri-Food Biotechnology Institute, Mohali, India. ${ }^{3}$ Centre for Genetic Manipulation of Crop Plants, University of Delhi South Campus, New Delhi, India.
}

Published online: 28 January 2020

\section{Reference}

1. Rajarammohan, et al. BMC Genomics. 2019;20:1036. https://doi.org/10.1186/ s12864-019-6414-6. 
A

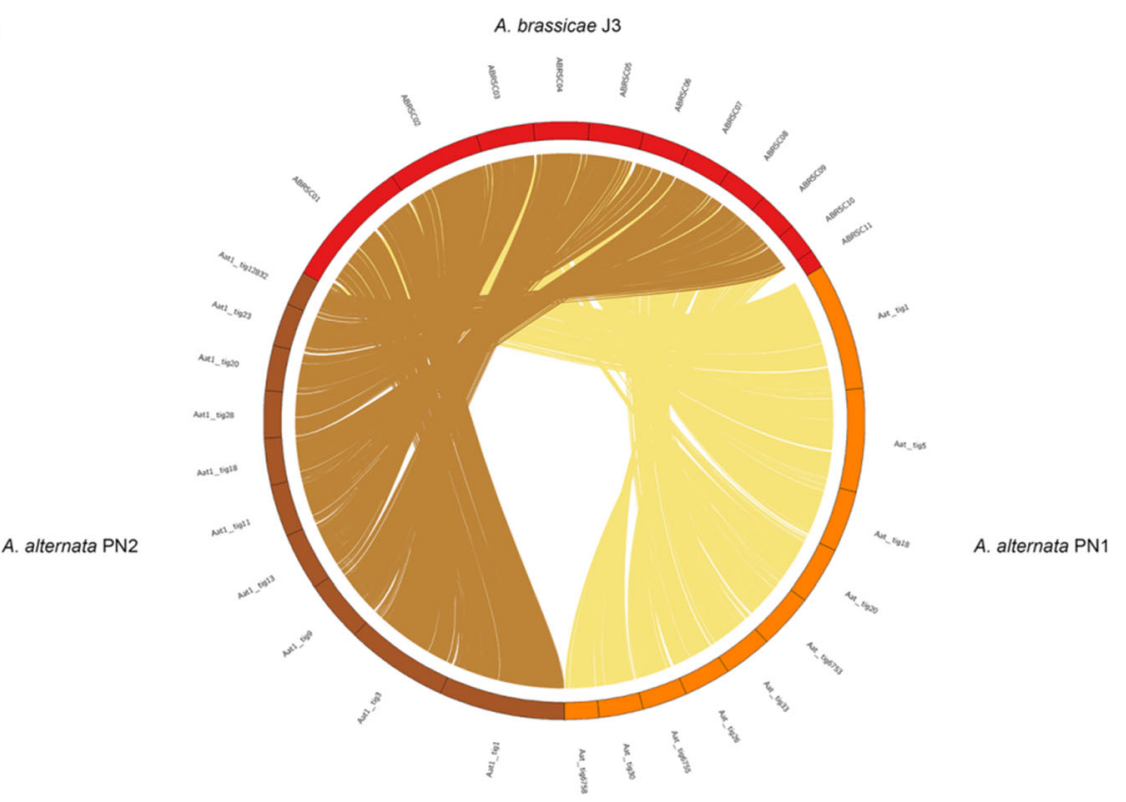

A. alternata PN1

A. alternata PN2

B

C

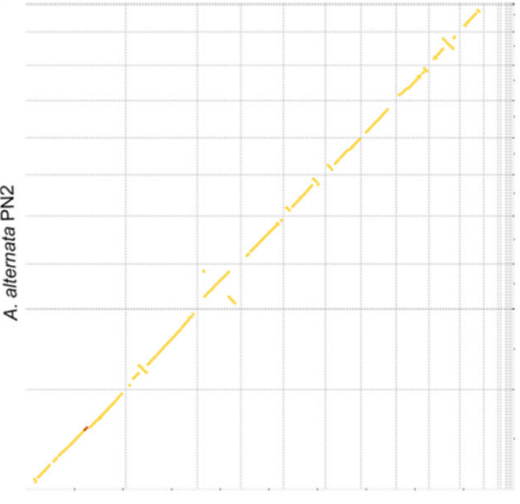

A. brassicae J3

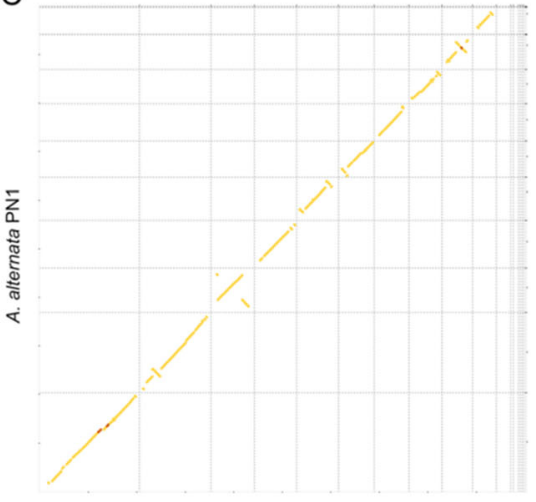

A. brassicae J3

Fig. 2 Whole-genome alignments of A. alternata PN1 and PN2 with A. brassicae. a Circos plot showing macrosynteny of A. alternata PN1 and PN2 with A. brassicae across all contigs except the dispensable contigs (ABRSC11, scaffold13,17,18,19), b and $\mathbf{c}$ Syntenic dotplots of $A$. brassicae with $A$. alternata PN1 and PN2 


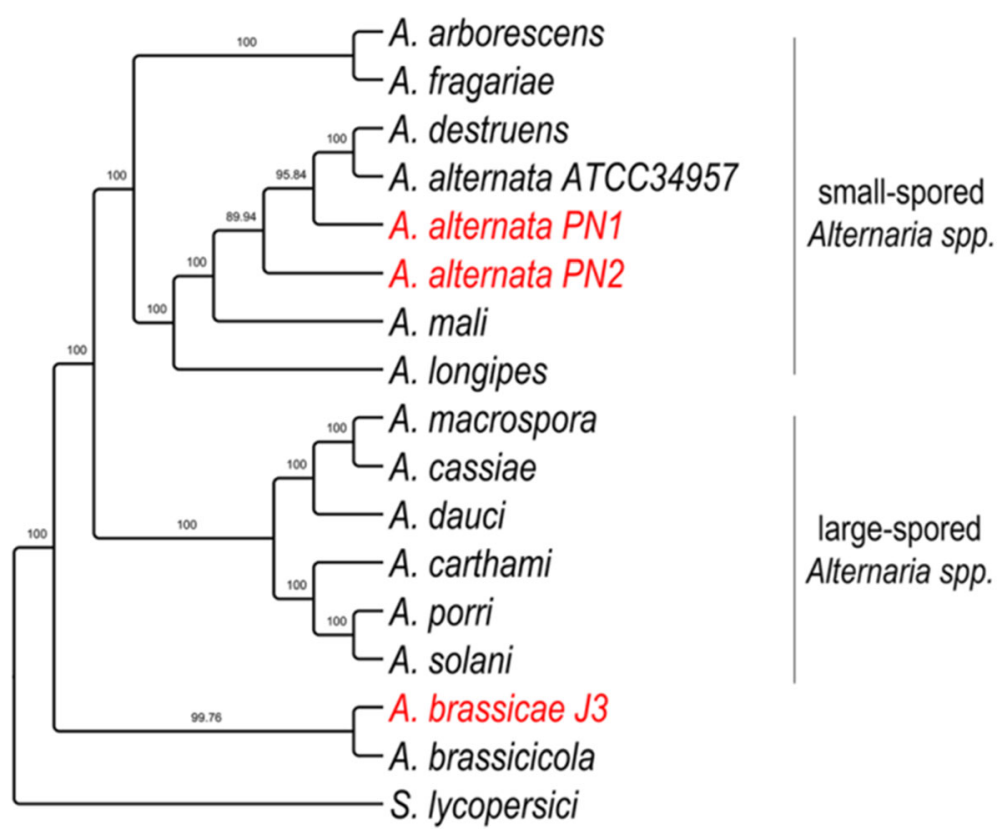

Fig. 3 Phylogenetic tree of Alternaria species with S. lycopersici as an outgroup. The tree was constructed using 29 single copy orthologs, which had the highest phylogenetic signal as calculated in Mirlo. Branch support values from 1000 bootstrap replicates are shown 